\title{
Clinico-Investigative Profile of Infantile Tremor Syndrome
}

\author{
Mahesh Kamate ${ }^{1}$
}

Received: 16 January 2020 / Accepted: 17 January 2020 / Published online: 27 January 2020

(C) Dr. K C Chaudhuri Foundation 2020

Developmental regression in a young child is always a challenge to investigate and treat [1]. Before we consider inherited metabolic conditions it is always important to rule out acquired conditions that are mostly treatable such as nutritional deficiencies like vitamin-B12 presenting as infantile tremor syndrome (ITS) and protein energy malnutrition; infections like human immunodeficiency virus and slow viral infections and environmental toxins like lead exposure etc.

Infantile tremor syndrome has been reported from India in the last six decades but the exact cause and treatment of this condition is not clear from the published literature [2]. Many studies done in the past have implicated many etiological factors like deficiency of zinc, vitamin-B12, iron, magnesium etc [3]. Role of vitamin-B12 deficiency in the causation of ITS is also not clear with many patients of ITS having normal vitamin-B12 levels. Is ITS really due to vitamin-B12 deficiency in these cases and if yes, how do work up these cases further is always a difficult task.

Many of us depend on the tremors to suspect and diagnose babies with ITS but it may not always be there in a given case and just regression of milestones with skin color change could be the only manifestations. Though previously termed as preITS in literature this has not been correctly characterized. Sankhyan and colleagues have done a detailed evaluation of 92 children and their mothers with ITS and pre-ITS using detailed clinical examination, standard psychometric tests, hematological tests and extensive biochemical tests to study the role of vitamin-B12 in the causation and treatment of ITS [4]. As presented in the study by Chaudhary et al., the tremors were seen only in $21.7 \%$ (20 out of 92) children and almost all of them had skin changes in the form of pallor and hyperpigmentation of skin. The absence of tremors in a given case

Mahesh Kamate

drmaheshkamate@gmail.com

1 Child Development and Pediatric Neurology Division, Department of Pediatrics, KAHER's J N Medical College,

Belgaum, Karnataka 590010, India may dissuade a physician from considering ITS in an infant with neuro-regression. The term Neurocutaneous infantile B12 (NIB) aptly describes the key clinical features, provides clue to the underlying cause and can lead to prompt treatment of this condition and there is a need to change from ITS/ preITS to neurocutaneous infantile vitamin B12 to label these children.

Serum vitamin-B12 levels were less than the normal cut off $(211 \mathrm{pg} / \mathrm{ml})$ in only $42 \%$ children in the current study which is similar to that reported by Goraya et al. [5]. The practice of many medical professionals prescribing multivitamin syrups as appetite stimulants could be one of the main reasons for getting normal vitamin-B12 levels in most of the cases. However other evidence of vitamin-B12 deficiency in the form of increased homocysteine levels in $96.5 \%$ cases, elevated $\mathrm{C} 3: \mathrm{C} 2$ ratio in $79.5 \%$ cases and increased urine methylmalonic acid in urine was seen in $70.1 \%$ cases. Only 3 out of the total 92 did not have any biochemical evidence of vitamin B-12 deficiency [4]. If we combine hematological findings, all children with ITS had some evidence of vitamin-B12 deficiency and none of them had folate deficiency in the study population.

As only $42 \%$ of cases had low serum vitamin-B12 levels, it would be a wise decision to do serum homocysteine levels in the baby if he has received vitamin-B12 supplementation in last few days. Estimating vitamin-B12 and homocysteine in the mother also is a good option if there is history of receiving oral vitamin-B12 for long time or if injectable vitamin-B12 was given to the baby in the past. As most of the babies are exclusively or predominantly breastfed, a low vitamin B12 in the mother would be a surrogate marker for vitamin-B12 levels in the child.

Majority of the children with ITS, are borne to exclusively breast-fed mostly vegan mothers who themselves are vitaminB12 deficient and because of delayed weaning the vitaminB12 deficiency in the baby gets exaggerated. Presentation with developmental delay and/or regression and response to vitamin-B12 supplementation confirms the role of vitaminB12 in the causation of ITS. If vitamin-B12 levels are normal in a given case of ITS, then other tests should be done. 


\section{Compliance with Ethical Standards}

Conflict of Interest None.

\section{References}

1. Clarke JTR. A Clinical Guide to Inherited Metabolic Disease, 2nd ed. Cambridge: Cambridge University Press; 2004.

2. Kaul KK. Infantile tremor syndrome: still a mystery! Indian Pediatr. 2015;52:628-31.
3. Agarwal R, Singh RN, Gupta BD, Agarwal DK, Arora AK. Serum magnesium, calcium, zinc in infantile tremor syndrome. Indian Pediatr. 1993;30:374-6.

4. Chaudhary H, Verma S, Bhatia P, Vaidya PC, Singhi P, Sankhyan N. Infantile tremor syndrome or a neurocutaneous infantile B12 deficiency (NIB) syndrome? Indian J Pediatr. 2020. https://doi.org/10. 1007/s12098-019-03117-w.

5. Goraya JS, Kaur S. Infantile tremor syndrome: a review and critical appraisal of its etiology. J Pediatr Neurosci. 2016;11:298-304.

Publisher's Note Springer Nature remains neutral with regard to jurisdictional claims in published maps and institutional affiliations. 